seem to be that anybody who performs an experiment with the clear expectation of a certain result, and then obtains that result, can only too easily fall into the trap of being less than fastidious in eliminating ambiguities of interpretation. The moral, for all scientists, is obvious.

\section{MOLECULAR LIQUIDS \\ Excitons Found?}

from our Molecular Physics Correspondent

ONE of the more notable received ideas to be dispelled in recent years, as far as the physics of condensed phases is concerned, is that the existence of localized electronic excitations, or "excitons", is an essentially solid state phenomenon conditional upon the regularity and symmetry of the crystal lattice. It was not until 1966 that Rice and Jortner (J. Chem. Phys., 44, 4470; 1966) pointed out the obvious, namely that, with respect to distances comparable with the wavelength of the exciting radiation, simple liquids also show the required translational symmetry-in the precise technical sense that a quasi-free electron may be represented as a plane wave to an accuracy effectivcly unimpaired by the fluctuations in liquid structure over distances of a few atomic radii.

Almost simultaneously, Beaglehole in Chicago (Phys. Rev. Lett., 15, 551; $1965)$ obtained spectra from liquid xenon which were consistent with the existence of Wannier-type excitons, those with relatively large electron-hole separation and correspondingly weak binding. Since then the experimental side of the field seems to have been relatively static, although a burst of considerable energy was registered as Rice, Jortner and colleagues began to extend their original theoretical insights. (See, for example, Rice, Nicolos and Jortner, J. Chem. Phys., 48, 2484; 1968; Fisher and Rice, Phys. Rev., 176, 409 ; 1968.)

A new paper now raises the question of whether excitons can be observed in molecular, as opposed to atomic, liquids. Eckhardt and Nichols (Phys. Rev. Lett., 29, 1221 ; 1972) of the University of Nebraska describe measurements of the specular reflexion spectrum of pure liquid $\alpha$-methylnaphthalene, in the region 215 to $400 \mathrm{~nm}$, which show both the broadening and redshift, relative to the corresponding spectrum in solution, characteristic of exciton formation. The comparison is not straightforward, however, because the opacity of the neat liquid in the required region is far too high for a detailed spectrum to be observed directly in absorption-hence the need to rely on reflexion spectra from the liquid surfacc. To effect the comparison it is then necessary to perform a Kramers-Krönig transform on the results, thus extracting the required imaginary part of the refractive index, which corresponds to the absorption.

The authors are cautious in their ascription of the results to exciton formation, pointing out that dielectric effects in the solutions used might lead to the observed shifts and broadening, although this seems somewhat unlikely.

Nevertheless, these doubts about interpretation reflect the extent of the difficulty of establishing real contact between quantum mechanical theories of the exciton phenomenon and the optical effects which can be reliably observed. Although valuable progress has been made by the Chicago school in relating exciton scattering, and hence lifetimes and band-broadening, to simple models for the interaction in the liquid, several largely independent difficulties-for example, uncertainty about the detailed nature of small-wavelength fluctuations in the liquid, and lack of information about the distribution of excited states in even the simplest substances--combine to frustrate most attempts to calculate quantitative broadenings and shifts from fundamental quantum statistical mechanics. The advances made are nonetheless considerable, for they have established the general interrelationships between the phenomenon and other questions of vital interest such as the dynamics of polarization waves and the characteristics of translational collective modes; the latter were introduced into the more general context of liquid theory by Zwanzig (Phys. Rev., 156, $190 ; 1967$ ).

For the time being the outlook would seem to favour the view, recommended by $\mathrm{Rice}$, that once liquid exciton formation is established it should be regarded as a particularly sensitive optical probe complementary to others now available for deriving information about time- dependent liquid structure, rather than as a problem demanding immediate elucidation through imperfect models of the liquid state. Of course, the distinction is not clear, and it is precisely for this reason that the problem is fascinating; to an extent rather unusual among similar problems, the whole verification of the construction placed upon the experimental results, and not simply their direct explanation, is at stake in the search for an adequate theoretical model. The resulting mix of experimental and theoretical difficulties is, at all events, extraordinarily rich, made up of unsolved, yet promising, problems in spectroscopy, $N$ body theory, and dielectric phenomena, to name only the principal ingredients.

\section{SEISMOLOGY \\ SHOAL versus Fallon}

from our Geomagnetism Correspondent

Although attempts to discriminate between earthquakes and underground nuclear explosions were given significant stimulus and emphasis by the political events of the 1960 s, they were necessarily firmly rooted in academic geophysics. In recent years, however, published comparisons of the effects of explosions and earthquakes seem to have acquired an altogether more explicitly academic flavour. Clearly the two aims are far from mutually exclusive; but it is perhaps indicative of a change in emphasis that in a new study of elastic wave radiation from the two types of event Lambert et al. (Geophys. $J ., 29,403$; 1972) barely mention discrimination. Indeed, they are far more interested in "information about the intrinsic character of the source and the propagation medium" and parameters "of fundamental geophysical interest".

\title{
Methane and Carbide on the Moon
}

IN next Monday's Nature Physical Science (January 22), Cadogan, Eglinton, Maxwell and Pillinger report measurements of the methane and carbide contents of Apollo 11 fines. Cadogan et al. took samples containing sizes between 108 and $152 \mu \mathrm{m}$ and separated them into six fractions with differing densities and magnetic susceptibilities. They find that both methane and carbide occur preferentially in those fractions which contain strongly magnetic particles, and they go on to explain this in terms of the exposure and impact histories of the individual grains.

Previous work by this Bristol group has shown that the contents of methane and carbide in lunar fines are closely related to the time for which the fines have been exposed on the lunar surface, and also that both compounds seem to reside chiefly on the surfaces of the particles. Their new measurements cause them to come out against one of the possible sources of carbide-an impact vapour cloud rich in iron and carbon. If much of the carbide in the fines were formed in this way, they say, then all particles should have a film of iron and carbide on them, and should thus be magnetic and contain comparable quantities of carbide. That is not the case.

On the other hand Cadogan et al. find in favour of carbide generation either by meteorite impact or solar wind implantation. Iron silicates could be reduced to iron under these circumstances and carbon already present in the particle could then be dissolved to form carbide. 